\title{
Acceptor and donor ionization energy levels in O-doped ZnTe
}

\author{
C. Tablero \\ Instituto de Energía Solar, Universidad Politécnica de Madrid, Ciudad Universitaria s/n, 28040 Madrid, Spain
}

A R T I C L E I N F O

Keywords:

Ionization levels

ZnTe:O

Intermediate band

Photovoltaics

\begin{abstract}
A B S T R A C T
The O-doped $\mathrm{ZnTe}\left(\mathrm{ZnTe}_{1}{ }_{x} \mathrm{O}_{x}\right)$ alloys present induce levels through $\mathrm{O}$ doping into the host semiconductor gap. ZnTe usually crystallizes in the zinc-blend structure and $\mathrm{ZnO}$ in the wurtzite structure under normal conditions. Therefore two possible $\mathrm{ZnTe}_{1} \mathrm{O}_{x}$ phases may coexist, although in different proportions, depending on experimental growth conditions. We present total energy calculations and analyze some of their electronic properties with respect to: the two ordered wurtzite and zinc-blende structures, the concentration ( $x$ from 0.0078 to 0.5 ), the localized basis set (from single-zeta to quadruple-zeta with polarization basis sets), and the variation of the ionization levels with $x$ and with the distance $\mathrm{O}-\mathrm{Zn}$.
\end{abstract}

\section{Introduction}

II-VI semiconductors have a potential for a variety of applications, especially as light-emitting and light-detecting devices, and solar cells. O-doped ZnTe compounds have very recently attracted the attention of researchers as intermediate-band solar cells, and samples with a high $O$ concentration have been grown $[1,2]$. A room-temperature photoluminescence spectrum for $\mathrm{ZnTe}: \mathrm{O}$ indicate $[1,2]$ a strong sub-bandgap response related to oxygen doping in the range of $1.6-2.0 \mathrm{eV}$. The strong emission for ZnTe:O denotes a highly radiative transition [1]. This sub-bandgap optical absorption increases as the oxygen increases, and is attributed to oxygen defects in ZnTe. The $0.4-0.5 \mathrm{eV}$ states below the conduction band (CB) act as an intermediate band (IB) for photovoltaic solar cells. The observed response for monochromatic $650 \mathrm{~nm}(1.91 \mathrm{eV})$ illumination suggests that carriers are excited from the valence band (VB) to the IB, and are then promoted to the $\mathrm{CB}$ by a further $650 \mathrm{~nm}$ photons, thermionic emission, or tunneling. The $\mathrm{ZnTe}$ diodes did not exhibit any detectable response at these wavelengths. The simultaneous excitation with $1550 \mathrm{~nm}(0.8 \mathrm{eV})$ and $650 \mathrm{~nm}(1.91 \mathrm{eV})$ laser sources results in an increase in photocurrent and open circuit voltage. The photovoltaic response increases monotonically by increasing the $1550 \mathrm{~nm}$ power density. It indicate that a two-photon process may be occurring in the ZnTe:O device [1]. The increase in the short circuit current by increasing the $1550 \mathrm{~nm}$ illumination suggests that the IB solar cell efficiency may be improved under higher excitation levels, indicating that these devices may further benefit from solar concentration.
The maximum power conversion limitation for single-junction solar cells is because the loss of photons with energy below the bandgap energy, and the low efficiency conversion of photons with energy exceeding the bandgap energy. The presence of the IB allows photons of lower energy than those of the gap to be absorbed, promoting electrons from the $V B$ to the $I B$, thus generating holes in the VB, and from the IB to the $\mathrm{CB}$, generating electrons in the $\mathrm{CB}$. In addition to this process of carrier generation, the usual generation process through photon absorption, promoting electrons from the $\mathrm{VB}$ to the $\mathrm{CB}$, also takes place. Therefore, the presence of the IB would increase the efficiency of solar cells, provided no voltage loss compensates for the current gain [3]. These materials have been studied in some detail during recent years [4-9], and several approaches have been proposed to make an IB practically, including quantum dots $[4,5]$, and doping with a high impurity concentration $[7,8]$. Quantum dots have demonstrated the intermediate band concept, where sub-bandgap response and increased efficiency have been reported [9]. In doped semiconductors, the properties may vary from localized defect states at low concentration to the formation of an energy band at high concentration. The former corresponds to impurity-doping whereas the later corresponds to impurity-alloying. For the impurity-alloying case, in order to form a band, the impurity concentration should exceed the threshold set by the Mott's transition $\left(\sim 10^{19} \mathrm{~cm}^{-3}[10]\right)$. This approach has been studied $[7,8]$ both theoretically and experimentally.

Because of the technological importance of the $\mathrm{ZnTe}_{1-x} \mathrm{O}_{x}$ alloys, it is of much interest to explore their electronic properties. It is experimentally difficult to control the phase and impurity concentration of the samples. In general, there are two phases in the experimental samples, although in different proportions. Therefore, it will very useful for optoelectronic applications to know 
the differences between some of the properties of both, the zincblende structure (ZBs) and the wurtzite structure (Ws), in the wide $x$ range. In this work, we report the results of first principles theoretical calculations for the acceptor and donor ionization levels of the $\mathrm{ZnTe}_{1_{-x}} \mathrm{O}_{x}$. We study both the Ws and the ZBs with $x$ from 0.0078 to 0.5

\section{Calculations}

Ionization energies are usually characterized by Kohn-Sham or Hartree-Fock defect levels (single-particle picture). But the singleparticle eigenvalues associated with the defect states do not account for the excitation aspect inherent to the ionization or recharging of the defect. The ionization or transition energies provide more rigorous information, since they are calculated from the total energies of the systems. Therefore, we use total energies in order to obtain the ionization energies.

The formation energy needed to incorporate one negatively charged $A$ atom into $M$ place, and thus form an acceptor $A$, in the MX semiconductor is $\Delta H_{f}\left(\mathrm{~A}_{\mathrm{M}}^{-}\right)=E\left(\mathrm{~A}_{\mathrm{M}}^{-}\right)-E_{H}-\mu_{A}+\mu_{M}-E_{F}$, where $E\left(\mathrm{~A}_{\mathrm{M}}^{-}\right)$denotes the total incorporation energy, $E_{H}$ is the total energy of the binary MX host, $E_{F}$ is the Fermi energy, $\mu_{A}$ and $\mu_{M}$ denote chemical potentials of the acceptor $\mathrm{A}$ and of the component $\mathrm{M}$ in the host semiconductor MX, respectively. These chemical potentials represent the energy of the reservoirs with which atoms are being exchanged. Therefore the incorporation of an $A_{M}^{-}$acceptor will be favored if more $A$ atoms are available (higher $\mu_{A}$ ), if the position of $E_{F}$ is higher (more energy is liberated due to the transfer of an electron from $E_{F}$ to $A$ ) and if more $M$ places are available (lower $\left.\mu_{M}\right)$. Similarly, the formation energy to incorporate one donor is $\Delta H_{f}\left(D_{\mathrm{M}}^{+}\right)=E\left(D_{\mathrm{M}}^{+}\right)-E_{H}-\mu_{D}+\mu_{\mathrm{M}}+E_{\mathrm{F}}$. The acceptor energy corresponds to the value of $E_{F}$ at which the impurity or the defect change from $A_{M}^{-}$to $A_{M}$, i.e. $e_{A}=E\left(A_{M}^{-}\right)-E\left(A_{M}\right)$. Similarly, the donor energy is $e_{D}=E\left(D_{M}\right)-E\left(D_{M}^{+}\right)$. For the host semiconductor, when $\mathrm{D}=\mathrm{A}=\mathrm{M}$, the acceptor and donor energies correspond with the $\mathrm{CB}$ and $\mathrm{VB}$ edge energies, i.e. $e_{\mathrm{C}}$ and $e_{V}$, and the gap is $E_{g}=e_{C}-e_{V}=E\left(M_{\mathrm{M}}^{-}\right)-2 E_{H}+E\left(M_{\mathrm{M}}^{+}\right)$.

The total energy of the aforementioned systems have been obtained with the density-functional theory (DFT) [11] using supercells containing from 32 to 256 atomic sites $\left(\mathrm{ZnTe}_{1-x} \mathrm{O}_{x}\right.$ with $x$ from 0.0078 to 0.5 ). The standard Kohn-Sham (KS) [12] equations are solved self-consistently [13]. For the exchange and correlation term the local-spin density approximation (LDA) and the generalized gradient approximation (GGA) have been used: the LDA with the Perdew-Zunger parametrization to the Ceperley-Alder numerical data [14], and the GGA in the form of Perdew et al. [15]. The standard Troullier-Martins [16] pseudopotential is adopted and expressed in the Kleinman-Bylander [17] factorization. The KS orbitals are represented using a linear combination of confined pseudoatomic orbitals [18]. Several further corrections to the calculated energies [19] were applied: potential alignment between a charged defect calculation and the perfect host crystal, and spurious interaction of periodic image charges.

An analysis of the basis set convergence has been carried out using from single-zeta to quadruple-zeta with polarization basis sets (SZP, DZP, TZP and QZP for single-, double-, triple- and quadruple-zeta with polarization) for all atoms and by varying the number of the special $k$ points in the irreducible Brillouin zone (BZ). The dimension of the $k$ point grid varies with the cell size in order to keep a constant $k$ point density in the BZ.

In order to study the $\mathrm{ZnTe}_{1-x} \mathrm{O}_{x}$ alloys we have used a large number of ordered structures (both ZBs and Ws) by varying the O compositions. For all of these structures we start from a unit cell where the $O$ atoms are replaced by Te. However, when $x$ increases, there are many non-equivalent atomic configurations. Therefore, we have used the more homogeneous structures for the calcula- tions. For the Ws, we have used 32-, 108- and 256-atom supercells. For the ZBs we have used 54-, 64- and 216-atom supercells. The number of atoms in the cell $(\mathrm{N})$ is related to the $\mathrm{O}$ concentration as $\sim 3,5.10^{22} / \mathrm{N} \mathrm{cm}^{-3}$ approximately.

For the supercells with a high $O$ concentration we have used the lattice parameters from the linear interpolation of the binaries for both, the ZBs and the Ws. ZnTe usually crystallizes in the ZBs under normal conditions. However, the Ws is structurally similar. The structural differences between the ZBs and the Ws refer only to the positions of the second neighbours. For ZnTe in the ZBs, the lattice parameter is $a=6.10 \AA$, the nearest-neighbour distance Te-Zn is $2.64 \AA$, and the direct gap is $2.4 \mathrm{eV}$. For the Ws, the lattice parameters are $a=4.27 \AA$ and $c / a=1.637$, and $r(\mathrm{Te}-\mathrm{Zn})=2.62 \AA[20]$. The $\mathrm{ZnO}$ is a wide band gap semiconductor of $3.4 \mathrm{eV}$. Its properties make it a candidate for various optoelectronic devices, such as visible and ultraviolet light emitters, transparent field-effect transistors, and laser diodes. Under ambient conditions, ZnO crystallizes with the Ws, but samples with ZBs have also been reported $[21,22]$. The lattice parameters for the Ws are $a=3.249 \AA$ and $c=5.205 \AA$ [23], and for the ZBs the lattice parameter is $a=4.58 \AA[22]$.

\section{Results and discussion}

\subsection{Modification of the ionization levels with the concentration}

Using total energy calculations and the structures described in the section II, we have obtained the ionization energies of the $\mathrm{ZnTe}_{1-x} \mathrm{O}_{x}$ alloys with $x$. The evolution of the acceptor and donor energies into a wide range of the concentration $x(0 \leqslant x \leqslant 0.5)$ are shown in Fig. 1 with the DZP and QZP basis sets, and for the Ws (panel a) and ZBs (panel b). With the TZP basis set the results are indiscernible with respect to the QZP basis set. For it, the results with the TZP basis set are not shown. By comparing the result with the DZP and QZP basis set, we see that the differences are very small. The energy origin of this figure corresponds to the ZnTe VB edge energy for both, the ZBs and the Ws. For $x=0$ the experimental gap for the ZBs is $2.4 \mathrm{eV}$. Therefore, the gap obtained with total energies, i.e. as differences between the host acceptor ( $C B$ edge)

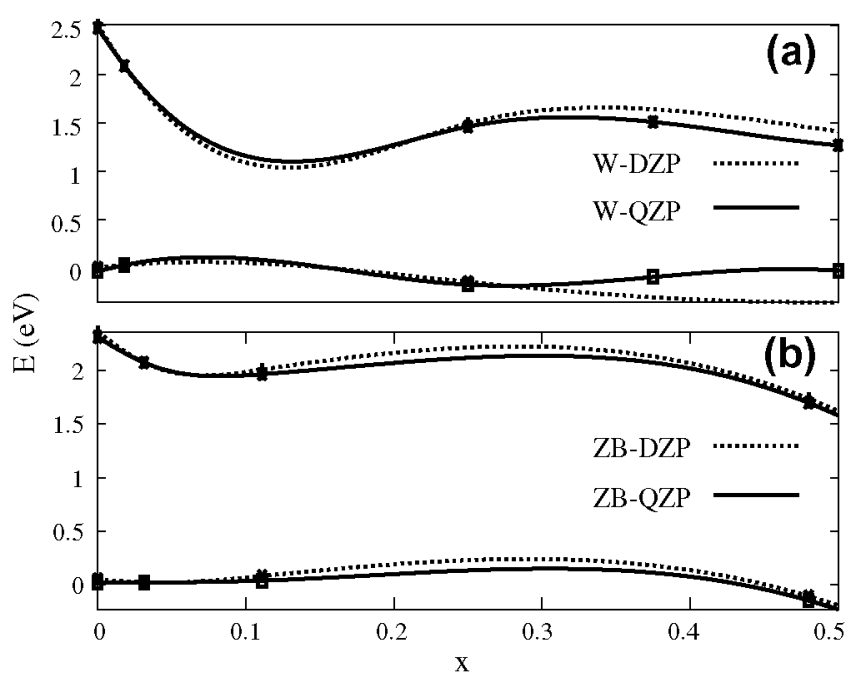

Fig. 1. $\mathrm{ZnTe}_{1-x} \mathrm{O}_{x}$ donor and acceptor energies using a DZP and QZP basis sets for the Ws (a) and ZBs (b) as a function of the $x$. The donor energy corresponds approximately to the VB energy. The top of the VB energy for ZnTe $(x=0)$ has been chosen as zero energy for the two structures. Marks indicate the calculated structures and lines correspond to fits to these points. 


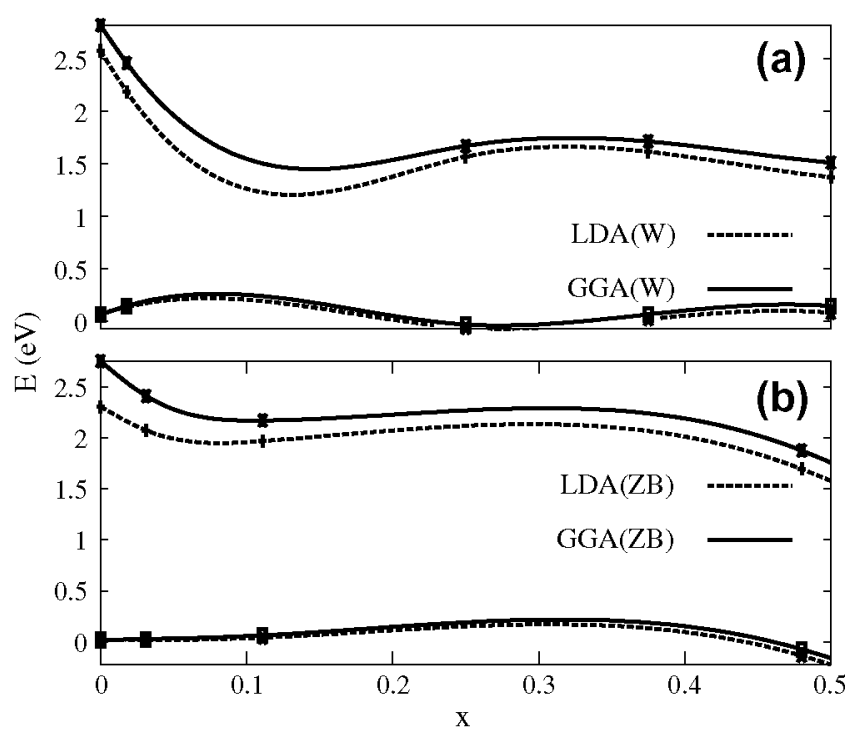

Fig. 2. $\mathrm{ZnTe}_{1-x} \mathrm{O}_{x}$ donor and acceptor energies using a TZP basis sets for the Ws (a) and ZBs (b) with LDA and GGA, as a function of the $x$. The top of the VB energy for $\mathrm{ZnTe}(x=0)$ has been chosen as zero energy for the two structures. Marks indicate the calculated structures and lines correspond to fits to these points.

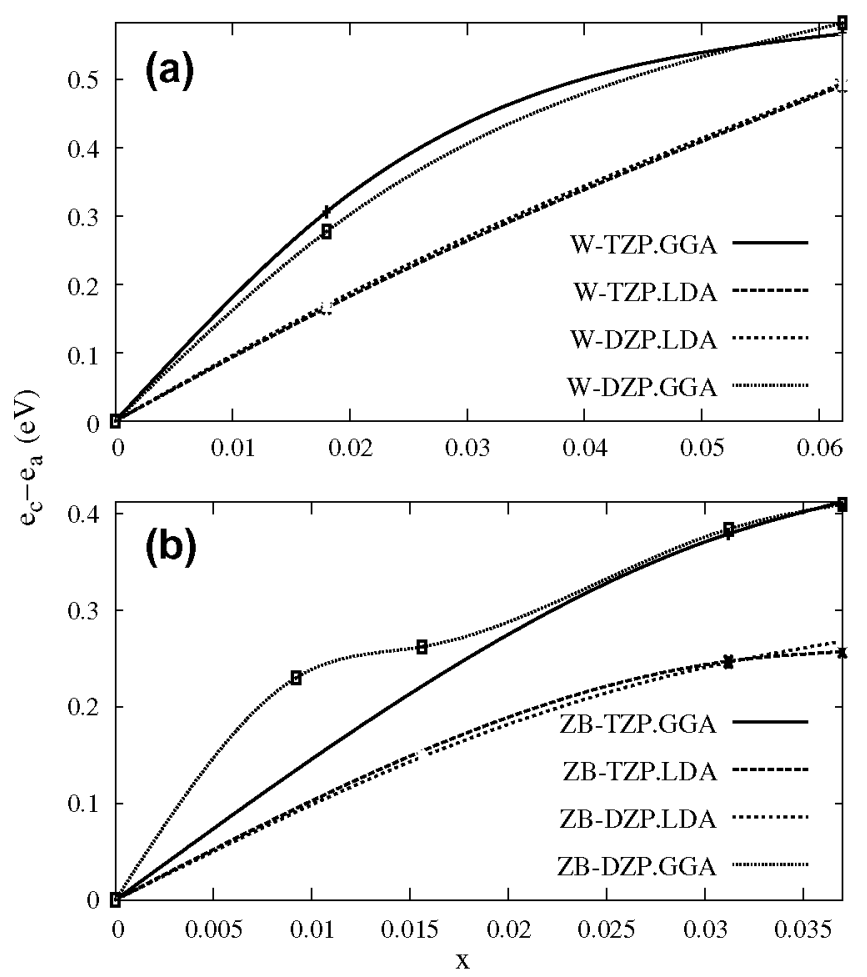

Fig. 3. Energy difference $e_{C}-e_{A}(\mathrm{eV})$ between the CB energy $e_{C}$ and the acceptor energy $e_{A}$ for $\mathrm{ZnTe}_{1-x} \mathrm{O}_{x}$ as a function of the $x$, using DZP and TZP basis sets, and LDA and GGA. Marks indicate the calculated structures and lines correspond to fits to these points.

energy and the host donor (VB edge) energy $\left(E_{g}=E\left(M_{\mathrm{M}}^{-}\right)-2 E_{\mathrm{H}}+\right.$ $E\left(M_{\mathrm{M}}^{+}\right)$), is similar to the experimental results for the ZBs (Fig. 1b). The gap for the Ws $(\sim 2.51 \mathrm{eV})$ is lightly larger than for the ZBs.

The differences between LDA and GGA using a TZP basis set on all atoms are represented in Fig. 2. The main difference between LDA and GGA is that with GGA the acceptor level is a little higher in energy. However, the CB edge with GGA is larger than with LDA. It can be seen in the Figure for $x=0$, where the host acceptor energy for ZnTe is the CB energy. Therefore, the final result when the acceptor level and the $\mathrm{CB}$ edge are analyzed simultaneously is that the acceptor energy for LDA is closer to the CB edge than with GGA. This is shown in Fig. 3 for a larger dilution and with different basis sets. In this figure the energy difference between the $\mathrm{CB}$ edge $\left(e_{C}\right)$ and the acceptor energy $\left(e_{A}\right)$ is represented using the largest supercells (lower $x$ ). These results compare well with the experimental [1] and theoretical single-particle [24] results in the literature, where the energy difference $e_{C}-e_{A}$ is around $0.4-0.5 \mathrm{eV}$.

This acceptor level is the origin of the IB into gap when the $O$ concentration increases. A deep level in the middle of the band gap is assumed to be the origin of non-radiative recombination (NRR). However, at high concentration the levels lead to bands, where NRR is reduced $[10,25]$. This fact could explain the high radiative transition for ZnTe:O observed experimentally [1].

\subsection{Change of the acceptor level with the distance $O-Z n$}

Furthermore, the energy difference $e_{C}-e_{A}$ and $e_{D}-e_{V}$ has been obtained as a function of the inward and outward displacement of the $\mathrm{O}-\mathrm{Zn}_{1}$ distance. It is a breathing-mode, i.e. the simplest type of localized vibration with spherical symmetry. The distance $\mathrm{O}-\mathrm{Zn}_{1}$ is chosen as a generalized coordinate $Q$. The changes in the acceptor and donor energies with respect to $Q-Q_{0}$, where $Q_{0}$ is the equilibrium experimental distance $\mathrm{Te}-\mathrm{Zn}$ in the host semiconductor, are shown in Fig. 4. From this Figure, an inward displacement $\left(Q-Q_{0}<0\right)$ increases the energy difference between the acceptor and the $\mathrm{CB}$ energy, causing the $e_{\mathrm{A}}$ level to go deeper into the gap.
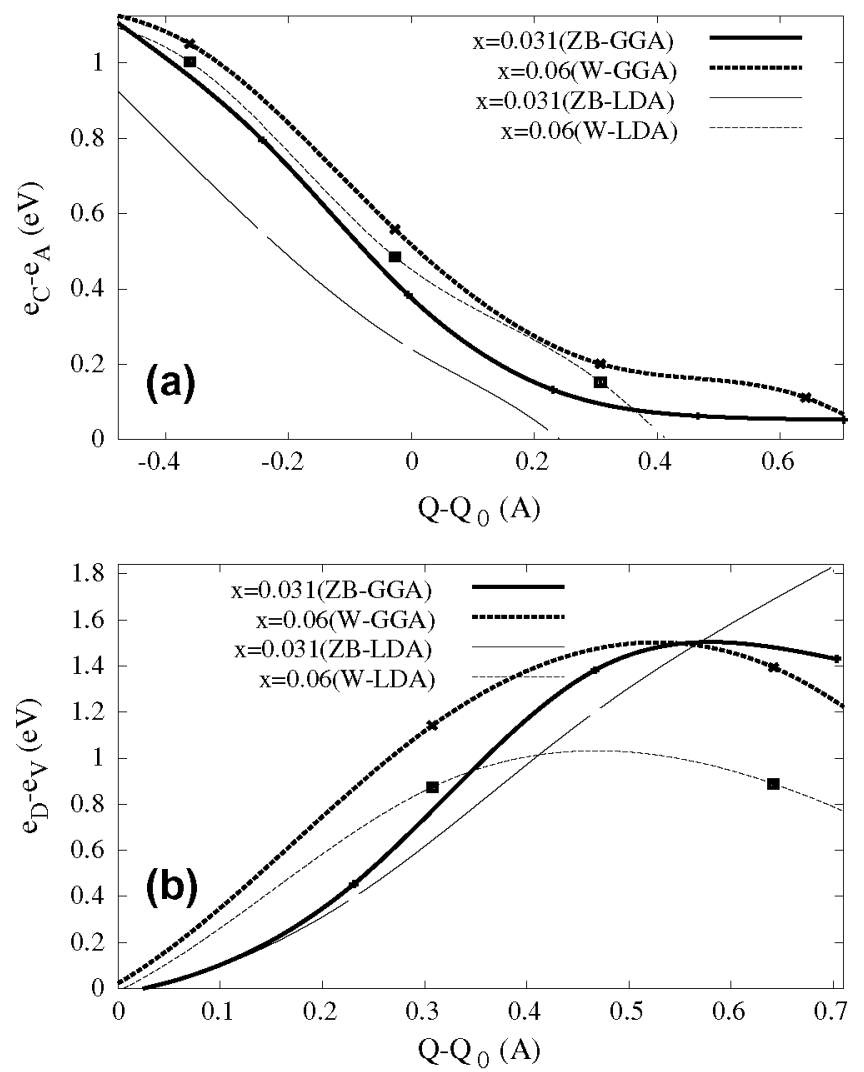

Fig. 4. Energy difference $(\mathrm{eV}) e_{C}-e_{A}$ (a) and $e_{D}-e_{V}$ (b) as a function of $Q-Q_{0}$ where $Q$ is the distance $O-Z_{1}(Q)$, and $Q_{0}$ is the equilibrium experimental distance $\mathrm{Te}-\mathrm{Zn}$ in the host semiconductor. Marks indicate the calculated structures and lines correspond to fits to these points. 
The main reason for this behavior is that the $e_{A}$ decreases more rapidly than $e_{C}$ when $Q-Q_{0}<0$. Therefore, with hydrostatic pressure this acceptor level is deeper in the gap. An outward displacement $\left(Q-Q_{0}>0\right)$ increases the energy difference between the donor and VB energy (Fig. $4 \mathrm{~b}$ ). As in the previous case, the change of the donor energy is larger than the variation of the VB energy with $Q-Q_{0}$.

\section{Conclusions}

In summary, we report the results for donor and acceptor energies of the doped $\mathrm{ZnTe}_{1-x} \mathrm{O}_{x}$ using first principles total energy calculations. Our results, with a wide range of $x$, compare GGA and LDA exchange and correlation terms, and different basis sets size, from SZP and QZP. These results compare well with experimental results in the literature. With GGA the gaps, and the energy differences $e_{C}-e_{A}$ and $e_{D}-e_{V}$ are larger than when LDA is used. The acceptor level is in the gap, whereas the donor level is very closer to the $\mathrm{VB}$.

We have also analyzed the dynamic evolution of the valence, donor, acceptor and conduction energies as a function of the generalized coordinate corresponding to a breathing-mode $\left(\mathrm{O}-\mathrm{Zn}_{1}\right.$ distance). The main effects are to displace the acceptor and donor levels toward the center of the gap with an inward and an outward displacement respectively, increasing $e_{C}-e_{A}$ and $e_{D}-e_{V}$.

\section{Acknowledgments}

This work has been supported by the GENESIS FV project of the National Spanish program CONSOLIDER (CSD2006-0004), by the European Commission through the funding of the project IBPOWER (Ref. N: Grant Agreement 211640), and by La Comunidad de Madrid through the funding of the project NUMANCIA-2 (Ref. N: S2009/ENE-1477).

\section{References}

[1] A.S. Lin, W. Wang, J.D. Phillips, J. Appl. Phys. 105 (2009) 064512.

[2] Y. Burki, P. Sshwendimann, W. Czaja, H. Berger, J. Phys.: Condens. Matter 5 (1993) 9235;

Y. Burki, P. Sshwendimann, W. Czaja, H. Berger, Europhys. Lett. 13 (1990) 555; V. Slusarenko, Y. Burki, W. Czaja, H. Berger, Phys. Status Sol. B 161 (1996) 897.

[3] A. Luque, A. Martí, Phys. Rev. Lett. 78 (1997) 5014.

[4] A. Martî, L. Cuadra, A. Luque, IEEE Trans. Electron. Devices 48 (2001) 2394; L. Cuadra, A. Martí, A. Luque, Physica E 14 (2002) 162; A. Martî, L. Cuadra, A. Luque, Physica E 14 (2002) 150;

A. Martí, L. Cuadra, A. Luque, IEEE Trans. Electron. Devices 49 (2002) 1632 A. Martí, E. Antolín, E. Cánovas, N. López, P.G. Linares, A. Luque, C.R. Stanley, C.D. Farmer, Thin Solid Films 516 (2008) 6716;
E. Cánovas, A. Martí, N. López, E. Antolín, P.G. Linares, C.D. Farmer, C.R. Stanley, A. Luque, Thin Solid Films 516 (2008) 6943.

[5] C.D. Cress, S.M. Hubbard, B.J. Landi, R.P. Raffaellea, D.M. Wilt, Appl. Phys. Lett. 91 (2007) 183108;

R. Oshima, A. Takata, Y. Okada, Appl. Phys. Lett. 93 (2008) 083111;

A. Alguno, N. Usami, T. Ujîhara, K. Fujiwara, G. Sazaki, K. Nakajima, Y. Shiraki, Appl. Phys. Lett. 83 (2003) 1258;

A.J. Nozik, Physica E 14 (2002) 115.

[6] K.M. Yu, W. Walukiewicz, J.W. Ager, D. Bour, R. Farshchi, O.D. Dubon, S.X. Li I.D. Shatp, E.E. Haller, Appl. Phys. Lett. 88 (2006) 092110; W. Wang, A.S. Lin, J.D. Phillips, Appl. Phys. Lett. 95 (2009) 011103 ; S. Suraprapapich, S. Thainoi, S. Kanjanachuchai, S. Panyakeow, Sol. Energy Mater. Sol. Cells 90 (2006) 2968.

[7] C. Tablero, Theor. Chem. Acc. 125 (2010) 23; C. Tablero, J. Appl. Phys. 106 (2009) 073718;

A. Martí, C. Tablero, E. Antolín, A. Luque, R.P. Campion, S.V. Novikov, C.T. Foxon, Sol. Energy Mater. Sol. Cells 93 (2009) 641; C. Tablero, A. Martí, A. Luque, J. Appl. Phys. 105 (2009) 033704;

C. Tablero, Comput. Mater. Sci. 44 (2008) 303;

C. Tablero, J. Phys.: Condens. Matter 19 (2007) 466209;

C. Tablero, J. Chem. Phys. 126 (2007) 164703;

C. Tablero, Solid State Commun. 143 (2007) 399;

C. Tablero, Phys. Rev. B 74 (2006) 195203;

C. Tablero, Solid State Commun. 133 (2005) 97;

C. Tablero, Phys. Rev. B 72 (2005) 035213.

[8] E. Cánovas, A. Martí, A. Luque, W. Walukiewicz, Appl. Phys. Lett. 93 (2008) 174109;

K.M. Yu, W. Walukiewicz, J. Wu, W. Shan, J.W. Beeman, M.A. Scarpulla, O.D. Dubon, P. Becla, Phys. Rev. Lett. 91 (2003) 246403;

K. Alberi, K.M. Yu, P.R. Stone, O.D. Dubon, W. Walukiewicz, T. Wojtowicz, X. Liu, J.K. Furdyna, Phys. Rev. B 78 (2008) 075201;

K. Alberi, J. Blacksberg, L.D. Bell, S. Nikzad, K.M. Yu, O.D. Dubon, W. Walukiewicz, Phys. Rev. B 77 (2008) 073202.

[9] A. Luque, A. Martî, N. López, E. Antolín, E. Cánovas, C. Stanley, C. Farmer, P. Díaz, J. Appl. Phys. 99 (2006) 094503;

A. Martî, E. Antolin, C.R. Stanley, C.D. Farmer, N. Lopez, P. Diaz, E. Canovas, P.G. Linares, A. Luque, Phys. Rev. Lett. 97 (2006) 247701.

[10] A. Luque, A. Martí, E. Antolín, C. Tablero, Physica B 382 (2006) 320.

[11] P. Hohenberg, W. Kohn, Phys. Rev. B 136 (1964) 864.

[12] W. Kohn, L.J. Sham, Phys. Rev. 140 (1965) A1133-A1138.

[13] J.M. Soler, E. Artacho, J.D. Gale, A. García, J. Junquera, P. Ordejon, D. SánchezPortal, J. Phys.: Condens. Matter 14 (2002) 2745. and references therein.

[14] J.P. Perdew, A. Zunger, Phys. Rev. B 23 (1981) 5048; D.M. Ceperley, B.J. Alder, Phys. Rev. Lett. 45 (1980) 566

[15] J.P. Perdew, K. Burke, M. Ernzerhof, Phys. Rev. Lett. 77 (1996) 3865; J.P. Perdew, K. Burke, M. Enzerhof, Phys. Rev. Lett. 78 (1997) 1396

[16] N. Troullier, J.L. Martins, Phys. Rev. B 43 (1991) 1993.

[17] L. Kleinman, D.M. Bylander, Phy. Rev. Lett. 48 (1982) 1425 ; D.M. Bylander, L. Kleinman, Phys. Rev. B 41 (1990) 907.

[18] O.F. Sankey, D.J. Niklewski, Phys. Rev. B 40 (1989) 3979.

[19] C. Persson, Yu-Jun Zhao, S. Lany, A. Zunger, Phys. Rev. B 72 (2005) 035211.

[20] O. Madelung, Semiconductors: Data Handbook, Springer, 2004.

[21] A.B.M. Almamun Ashrafi, A. Ueta, A. Avramescu, H. Kumano, I. Suemune, Y.W. Ok, T.Y. Seong, Appl. Phys. Lett. 76 (2000) 550.

[22] Shao-Min Zhou, He-Chun Gong, Bin Zhang, Zu-Liang Du, Nanotechnology 19 (2008) 175303.

[23] W-phase H. Karzel, W. Potzel, M. Köfferlein, W. Schissl, M. Steiner, U. Hiller, G.M. Kalvius, D.W. Mitchell, T.P. Das, P. Blaha, K. Schwarz, M.P. Pasternak, Phys. Rev. B 53 (1996) 11425.

[24] C. Tablero, A. Martí, A. Luque, Appl. Phys. Lett. 96 (2010) 121104.

[25] C. Tablero, Physica B 404 (2009) 4023. 